\title{
Mechanisms of brain injury in the newborn
}

\begin{abstract}
Advances in obstetric and neonatal medical care have led to marked improvements in the chances of survival for extremely preterm and low birth weight babies. This review focuses on the mechanisms of neurological injury in extremely preterm and critically ill infants and discusses current progress in therapeutic strategies.

Eye (2007) 21, 1261-1263; doi:10.1038/sj.eye.6702848
\end{abstract}

Keywords: human infant; perinatal brain injury; neuroprotection; visual impairment

\section{Introduction}

Advances in obstetric and neonatal medical care have led to marked improvements in the chances of survival for extremely preterm and low birth weight babies. However, there is continuing concern about the high rate of neurological, educational, and behavioural problems in survivors. In addition, acute perinatal asphyxia is an important cause of disabling brain injury in term infants. This short review will focus on the mechanisms of neurological injury in extremely preterm and critically ill infants undergoing intensive care and current progress in therapeutic strategies.

\section{Brain development}

The last 3 months of gestation represents a critical period in the development of the central nervous system. The process of neuronal migration within the cerebral hemispheres is largely complete by 22-24 weeks gestation. Neurons generated within the subependymal matrix of the lateral ventricles physically migrate through the substance of the cerebral hemisphere until they achieve their final position within the cerebral cortex. From 24 weeks of gestation until term, each cortical neuron will establish approximately 1000
JS Wyatt

synaptic connections, creating the great bulk of cortico-cortical connections within the cerebral hemispheres. This is sometimes described as the 'flowering' of the dendritic tree. It is estimated that there are approximately $10^{11}$ neurons and $10^{14}$ synapses within the human central nervous system. The majority of these connections are created in the last 3 months of pregnancy. A back of the envelope calculation gives the astonishing figure of several hundred million synapses created every minute.

\section{Magnetic resonance imaging}

Magnetic resonance imaging of extremely preterm infants has enabled the dramatic growth of the cortex and cerebral hemispheres to be visualised. ${ }^{1,2}$ At 25 weeks, the cortex appears smooth and featureless, the lateral ventricles are relatively large, and the white matter is thin. By term, the grey matter volume has increased four-fold and the surface area of the cortex has increased approximately eightfold. The major tracts of the white matter, especially the corpus callosum and its connections, increase substantially in volume and complexity over the same period.

In normal human development, the dramatic growth in brain connections takes place within the highly controlled and relatively sensorally deprived environment of the human uterus. Yet when an extremely preterm baby is delivered at 24 weeks gestation, this critical period of brain development takes place within the highly atypical environment of a neonatal intensive care unit. It is not surprising, therefore, that there is increasing evidence that the normal process of brain development is adversely affected by extremely preterm delivery.

\section{Evidence of brain abnormalities in preterm infants}

Magnetic resonance imaging studies of very preterm infants scanned at term have shown a

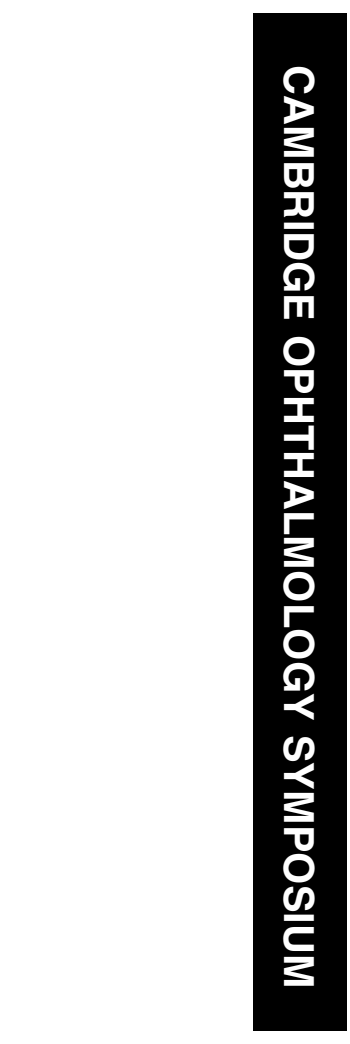

Perinatal Brain Research Group, University College London, London, UK

Correspondence: JS Wyatt, Perinatal Brain Research Group,

University College London, 5 University Street, London WC1E 6JJ, UK Tel: + 442076796113 ; Fax: +442076796103.

E-mail: john.wyatt@ uclh.org

Received: 4 March 2007 Accepted: 29 March 2007 
number of abnormalities. ${ }^{3,4}$ These include focal and generalised white matter abnormalities, impaired cortical folding, and reduced grey and white matter volumes. Reduced growth and development of the posterior corpus callosum and its connections seems to be a particularly common finding. Diffusion tensor imaging has also confirmed that impaired formation of major white matter tracts is common in ex-preterm infants.

\section{Mechanisms of white matter injury}

These findings emphasise the unusual predominance of white matter injury in very preterm infants. There appear to be several underlying factors that contribute to this phenomenon. Firstly, the arterial vascular supply of the immature brain leads to arterial end zones within the deep periventricular white matter. Secondly, the autoregulatory control mechanisms within the cerebral vasculature are immature. This implies that fluctuations in arterial blood pressure induced during intensive care may be transmitted directly to the cerebral blood vessels, causing marked variations in cerebral blood flow. As a consequence, specific regions within the deep white matter are likely to be highly vulnerable to ischaemic and haemorrhagic injury.

Thirdly, there is evidence of the selective vulnerability to injury of a population of immature glial cells, which are present in the periventricular white matter in the third trimester. In vitro studies of oligodendroglial precursor cells have indicated that they are extremely vulnerable to hypoxic-ischaemic injury. A range of pathogenic factors, including free radicals, glutamate, and pro-inflammatory cytokines, have all been shown to induce death of immature oligodendroglial cell lines. In addition, the presence of free iron following germinal matrix or intraventricular haemorrhage may exacerbate the sensitivity of oligodendroglial cells to free radical injury. The end result of these pathological processes is cystic tissue necrosis within the periventricular white matter regions and the development of periventricular leukomalacia (see Kapellou $e t a l^{5}$ for review).

Fourthly, very preterm infants have a prominent and highly vascular germinal matrix within the subependymal layers lining the lateral ventricles. This region is a frequent source of haemorrhage leading to the formation of blood clot within the lateral ventricles. In the most severe cases, a venous haemorrhagic infarct develops within the white matter adjacent to the lateral ventricle, leading ultimately to tissue necrosis and the development of a porencephalic cyst within the white matter.

Thus, a range of factors that are uniquely present in the very preterm infant lead to enhanced vulnerability of the cerebral white matter to injury.

\section{Brain injury in the term infant}

By contrast, a very different pattern of injury is seen in the term infant exposed to acute perinatal asphyxia. At this stage of brain development, it is the central grey matter regions, especially the basal ganglia and thalami, which are specifically vulnerable to hypoxic-ischaemic injury. In addition, cortical necrosis and subcortical white matter injury are commonly observed following acute asphyxia. It is likely that the very different pattern of brain injury reflects changes in brain maturation. In particular, there are enhanced metabolic demands within the central and peripheral grey matter, together with a marked increase in the density of glutamate receptors associated with synapse formation.

\section{Environmental influences}

While the very preterm infant is undergoing intensive care, the brain is exposed to a range of potentially damaging influences. There is continuous sensory bombardment from light, sound, and touch over many weeks. An array of unpleasant and noxious procedures such as arterial and venous cannulation, endotracheal intubation, and heel-prick blood sampling are performed. The effect of continuous sensory bombardment is to interfere with normal sleep-wake cycling including interference with rapid eye movement sleep. This may be particularly important in the development of normal cortical visual connections. Studies in newborn rodents have suggested that REM sleep may provide neuronal stimuli for shaping synaptic connections in visual pathways. In the newborn rodent, 7 days of REM sleep deprivation led to alterations in synaptic plasticity in the visual cortex. ${ }^{6}$ In addition, medications including analgesics, sedatives, and corticosteroids may all interfere with the normal processes of brain development and the development of synaptic connectivity. A further effect of the intensive care environment may be to obstruct the visual field and interfere with the development of binocular vision, through the presence of tubing and respiratory devices fixed to the infant's nose and forehead.

Increased awareness of the potentially adverse effects of the intensive care environment is leading to active exploration of alternative methods of caring for premature babies. One approach, described as individualised developmental care, is designed to minimise interference with the baby's own individual patterns and responses while intensive care is given. Preliminary studies have indicated that this approach, although requiring intensive staffing and education, may be associated with improved neurodevelopmental 
outcome and with an improvement in white matter imaging appearances and connectivity. ${ }^{7}$

\section{Compensatory mechanisms}

Despite the frequency of white matter injury in ex-preterm children, there appears to be a remarkable ability for the developing brain to compensate for structural abnormalities present from early life. Thus studies of ex-premature adolescents have shown that while many individuals have obvious white matter abnormalities on MRI scanning, a significant number appear to be functioning within the normal range with detailed neuropsychological and cognitive testing. ${ }^{8}$

A fascinating study by Santhouse et $a l^{9}$ gives insights into the possible mechanisms of adaptation. A group of ex-premature adults underwent structural MRI, and those individuals with posterior corpus callosal abnormalities were identified. The subjects then underwent a visual processing task, which involved the matching of symbols within opposite visual fields. Functional MRI was performed during the task. The primary hypothesis was that the subjects with corpus callosal abnormalities would demonstrate reduced abilities on the visual matching task compared with controls. Surprisingly, there was no significant difference between the groups. However, fMRI indicated that subjects with the corpus callosal abnormalities had a different activation pattern. In particular, activation of a region within the right dorsolateral prefrontal cortex was seen, a region previously associated with working visual memory. The investigators concluded that the subjects had developed a visual processing strategy for comparing items in the visual fields, which did not employ the corpus callosum.

A number of possible mechanisms may be used during brain development to compensate for injury sustained in the perinatal period. These include the remodelling of existing white and grey matter regions, the refinement and selection of dendritic connections, the rerouting of white matter tracts to circumvent obstructions, and the development of alternative cortical processing strategies.

\section{Conclusion}

These mechanisms offer a number of therapeutic targets for novel interventions and therapeutic programmes designed to improve outcome in infants following perinatal brain injury. It can be hoped that over the next decade, a range of new therapeutic programmes will be developed, using the rapidly accumulating knowledge about the inherent compensatory processes within the developing brain.

The author acknowledges the major contribution of colleagues at University College London, the Institute of Child Health and the Institute of Psychiatry, London.

\section{References}

1 Kapellou O, Counsell SJ, Kennea N, Dyet L, Saeed N, Stark J et al. Abnormal cortical development after premature birth shown by altered allometric scaling of brain growth. PLoS Med 2006; 3: e265.

2 Huppi PS, Warfield S, Kikinis R, Barnes PD, Zientara GP, Jolesz FA et al. Quantitative magnetic resonance imaging of brain development in premature and mature newborns. Ann Neurol 1998; 43: 224-235.

3 Inder TE, Warfield SK, Wang H, Huppi PS, Volpe JJ. Abnormal cerebral structure is present at term in premature infants. Pediatrics 2005; 115: 286-294.

4 Robertson NJ, Wyatt JS. The magnetic resonance revolution in brain imaging: impact on neonatal intensive care. Arch Dis Child Fetal Neonatal Ed 2004; 89: F193-F197.

5 Haynes RL, Baud O, Li J, Kinney HC, Volpe JJ, Folkerth DR. Oxidative and nitrative injury in periventricular leukomalacia: a review. Brain Pathol 2005; 15: 225-233.

6 Shaffery JP, Sinton CM, Bissette G, Roffwarg HP, Marks GA. Rapid eye movement sleep deprivation modifies expression of long-term potentiation in visual cortex of immature rats. Neuroscience 2002; 110: 431-443.

7 Als H, Duffy LH, McAnulty GB, Rivkin MJ, Vajapeyam S, Mulkern RV et al. Early experience alters brain function and structure. Pediatrics 2004; 113: 846-857.

8 Rushe TM, Rifkin L, Stewart AL, Townsend JP, Roth SC, Wyatt JS et al. Neuropsychological outcome at adolescence of very preterm birth and its relation to brain structure. Dev Med Child Neurol 2001; 43: 226-233.

9 Santhouse AM, ffytche DH, Howard RJ, Williams SC, Stewart $\mathrm{AL}$, Rooney $\mathrm{M}$ et al. The functional significance of perinatal corpus callosum damage: an fMRI study in young adults. Brain 2002; 125: 1782-1792. 